\title{
Optimal time interval from surgery to adjuvant chemotherapy in gastric cancer
}

\author{
GEON TAE AHN ${ }^{1}$, SUN KYUNG BAEK ${ }^{1,2}$, JAE JOON HAN ${ }^{1,2}$, \\ HONG JUN KIM ${ }^{1,2}$, SU JIN JEONG ${ }^{3}$ and CHI HOON MAENG ${ }^{1,2}$

\begin{abstract}
${ }^{1}$ Department of Internal Medicine, Kyung Hee University Hospital; ${ }^{2}$ Division of Medical Oncology and Hematology, Department of Internal Medicine, Kyung Hee University Hospital, Kyung Hee University College of Medicine;
\end{abstract} \\ ${ }^{3}$ Statistics Support Department, Medical Science Research Institute, Kyung Hee University Hospital, Seoul 02447, Korea
}

Received April 9, 2020; Accepted July 1, 2020

DOI: $10.3892 / \mathrm{ol} .2020 .11893$

\begin{abstract}
The effect of adjuvant chemotherapy (AC) for resected gastric cancer is well established; however, delays in treatment and its impact on clinical outcomes have not yet been determined. The current study analyzed the survival rates based on time interval (TI) between surgery and $\mathrm{AC}$ administration to evaluate a potential association between the two variables. Patients diagnosed with stage II-III gastric adenocarcinoma between 2009 and 2016 at the Kyung Hee University Hospital were included. Patients' data including demographics, TNM stage, types of AC, and TI retrospectively collected from surgery to the start of AC. Patients were dichotomized based on the TI, which was predetermined at $3,4,5,6,7$ or 8 weeks. Median disease-free survival (DFS) and overall survival (OS) were analyzed according to TI. In total, 172 patients were identified. The median follow-up duration was 40.8 (3-109) months. The median TI was 4.1 (2.1-9.8) weeks. DFS in patients with TI $\geq 4$ weeks $(n=106$, $61.6 \%$ ) was significantly lower compared with patients with TI $<4$ weeks $(n=66,38.4 \%)$, with a median DFS of TI $<$ vs. $\geq 4$ weeks of 8.1 vs. 6.0 years [hazard ratio $(\mathrm{HR})=1.80,95 \%$ confidence interval (CI): 1.067-3.045, $\mathrm{P}=0.0277]$. OS was also significantly reduced in patients with $\mathrm{TI} \geq 4$ weeks, favoring $\mathrm{TI}$ $<4$ weeks [median OS of TI < vs. $\geq 4$ weeks: Not reached (NR) vs. 7.0 years, $\mathrm{HR}=2.15,95 \% \mathrm{CI}$ : $1.173-3.939, \mathrm{P}=0.0133$ ]. Other predetermined TIs were not associated with survival outcomes. The current study demonstrated that $\mathrm{AC}$ within 4 weeks of surgery should be recommended for gastric cancer, and delays of $>4$ weeks may be detrimental to patients' survival.
\end{abstract}

Correspondence to: Dr Chi Hoon Maeng, Department of Internal Medicine, Kyung Hee University Hospital, Kyung Hee University College of Medicine, 23 Kyungheedaero, Dongdaemun-gu, Seoul 02447, Korea

E-mail:mchihoon@khu.ac.kr

Key words: gastric cancer, adjuvant chemotherapy, recurrence

\section{Introduction}

Gastric cancer is one of the most common cancers worldwide(1). Its incidence varies by region, with a high representation in East Asia, Eastern Europe, and South America. Surgery is currently the sole curative treatment option for patients with advanced gastric cancer; however, a substantial number of patients experience disease recurrence $(2,3)$. Adjuvant chemotherapy (AC) such as S-1 monotherapy or combination therapy with capecitabine and oxaliplatin (XELOX) has been the standard of treatment following gastrectomy with D2 dissection for pathologic stages II or III $(4,5)$. However, the optimal time for postoperative chemotherapy initiation after surgery is yet to be established. A significantly short time interval (TI) between surgery and AC can affect the patient's recovery from surgery and is more likely to cause problems including surgical wound complications. In contrast, a long TI between surgery and AC leads to a high risk of cancer recurrence due to growth of microscopic metastases. In the real world, delays in AC administration after surgery are common for various medical conditions such as postoperative complications, a decline in the patient's physical status as well as other nonmedical reasons such as low patient compliance, delayed consultation with medical oncologist, or economic issues $(6,7)$. In our hospital, AC is routinely administered within 4-6 weeks of surgery.

According to the 2017 Japan Gastric Cancer Guidelines, AC, particularly in the case of S-1 chemotherapy, is empirically recommended within 6 weeks of surgery (8). The Korean Practice Guidelines for Gastric Cancer 2018 were recently published following an evidence-based multidisciplinary approach, but did not include a recommended time point for the start of AC after gastrectomy (9). There were also no specific comments that focused on the time of $\mathrm{AC}$ administration in the pivotal phase 3 trials that have confirmed the role of $\mathrm{AC}$ in gastric cancer $(10,11)$. In a recent phase 3 trial evaluating the role of perioperative chemotherapy with or without immunotherapy, a TI for AC was defined at 4-10 weeks following surgery (12). Due to the fact that a precise cutoff value has not been established for the delayed time in AC administration, we analyzed the survival rate according to a predefined TI between surgery and the start of AC and attempted to define 
an optimal TI. In cases where delays were observed, we also addressed the reasons for this occurrence.

\section{Patients and methods}

Patients. Clinical data from patients diagnosed with stage II-III gastric adenocarcinoma who received AC after gastrectomy with D2 lymph node dissection between 2009 and 2016 in Kyung Hee University Hospital were reviewed retrospectively. Among these, patients eligible for analysis with accurate records were evaluated. Patients' data included demographics, TNM staging, types of chemotherapeutic agents, and TI between surgery and the start of AC. TI was defined as the period from the date of surgery to the start of AC. Staging of cancer was based on the guidelines established by the American Joint Committee on Cancer 7th edition. The protocol for the study was reviewed and approved by the Institutional Review Board (IRB) of Kyung Hee University Hospital (approval no. KHUH 2020-01-044). All analyses and writing of the manuscript were in accordance with the Declaration of Helsinki.

Data collection and statistical analyses. This study aimed to investigate the clinical effect of TI on disease recurrence and survival. Therefore, we first searched for variables that affected the clinical outcomes in these patients. We subsequently analyzed the impact of TI on disease recurrence and overall survival (OS) after adjusting for other variables using the Cox regression analysis. We investigated whether the initially planned AC was successfully completed, and reasons for delays in AC administration when TI was over 4 weeks were also determined. To compare the effect of TI between the two groups, the TIs were dichotomized based on the predetermined times of $3,4,5,6,7$, or 8 weeks and on the median value of TI for each patient. OS was defined as the period from the date of surgery to the last follow-up or death from any cause. Disease-free survival (DFS) was defined as the period from the date of surgery to the time of cancer recurrence or death from any cause. Survival outcomes were estimated using the Kaplan-Meier survival curves. A log-rank test was performed when a significant difference between the survival curves for each of the groups was observed. In case of the effect of the types of adjuvant chemotherapy on the survival outcomes, firth's penalized maximum likelihood bias reduction method for Cox regression was used. When statistically significant factors were observed in the univariate analysis, multivariate analysis using a Cox regression model were performed. A P-value $<0.05$ was considered statistically significant. All statistical analyses were performed using the Statistical Package for the Social Sciences version 23 package (International Business Machines Corporation) and R 3.5.3 software (https://cran.r-project.org).

\section{Results}

Patients characteristics. A total of 172 patients were identified in this study. Among these, 97 patients (56.4\%) were diagnosed with stage II gastric adenocarcinoma and 75 patients (43.6\%) with stage III gastric adenocarcinoma. All patients included in this study underwent surgical resection with
Table I. Patient characteristics.

\begin{tabular}{|c|c|c|}
\hline Variables & $\mathrm{N}$ & $\%$ \\
\hline \multicolumn{3}{|l|}{ Age (year) } \\
\hline Median (range) & 51.5 & $(21-82)$ \\
\hline$<60$ & 88 & 51.2 \\
\hline$\geq 60$ & 84 & 48.8 \\
\hline \multicolumn{3}{|l|}{ Sex } \\
\hline Male & 124 & 72.1 \\
\hline Female & 48 & 27.9 \\
\hline \multicolumn{3}{|l|}{ Stage } \\
\hline II & 97 & 56.4 \\
\hline III & 75 & 43.6 \\
\hline \multicolumn{3}{|c|}{ Type of adjuvant chemotherapy } \\
\hline XELOX & 48 & 27.9 \\
\hline TS-1 & 86 & 50.0 \\
\hline FP & 24 & 13.9 \\
\hline TS-1/Cisplatin & 6 & 3.4 \\
\hline FOLFOX & 5 & 2.9 \\
\hline Doxifluridine & 3 & 1.7 \\
\hline Median (range) & 4.1 & $(2.1-9.8)$ \\
\hline \multicolumn{3}{|l|}{ Recurrence } \\
\hline No & 104 & 60.4 \\
\hline Yes & 68 & 39.6 \\
\hline \multicolumn{3}{|c|}{ Pattern of recurrence $\mathrm{a}^{\mathrm{a}}$} \\
\hline Loco-regional & 6 & 8.9 \\
\hline Distant & 62 & 91.1 \\
\hline \multicolumn{3}{|l|}{ Death } \\
\hline No & 116 & 67.4 \\
\hline Yes & 56 & 32.6 \\
\hline \multicolumn{3}{|c|}{$\begin{array}{l}\text { Planned adjuvant chemotherapy } \\
\text { was completed }\end{array}$} \\
\hline No & 98 & 57.0 \\
\hline Yes & 74 & 43.0 \\
\hline Total & 172 & 100 \\
\hline
\end{tabular}

XELOX, oxaliplatin plus capecitabine; FP, cisplatin plus 5-fluorouracil; FOLFOX, oxaliplatin plus leucovorin plus

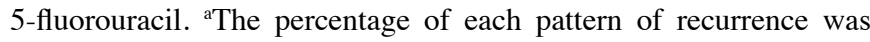
calculated based on the number of patients $(n=68)$ who had disease recurrence rather than the total number of the patient population $(n=172)$.

D2 lymph node dissection. The median age of the patient population was 51.5 years, with a higher proportion of male patients $(n=124,72 \%)$. Six types of chemotherapy were used as follows: XELOX (capecitabine and oxaliplatin), TS-1, FP (5-fluorouracil plus cisplatin), TS-1/Cisplatin, FOLFOX (oxaliplatin, leucovorin plus 5-fluorouracil) and doxifluridine in $27.9,50.0,13.9,3.4,2.9$, and $1.7 \%$ of patients, respectively. The median follow-up duration was 40.8 (range, 3-109) months. Recurrence was observed in a total of 68 patients (39.5\%), with most of the patients presenting with distant metastases $(n=62$ out of $68,91.1 \%)$. Patients' characteristics are summarized in 


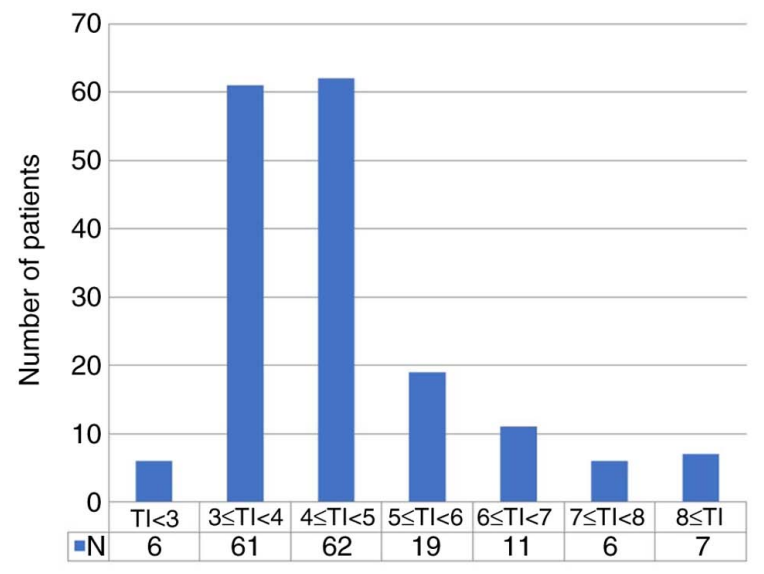

Figure 1. Patients' distribution according to time interval. TI, time interval; $\mathrm{N}$, number of patients.

Table I, and TI distribution is shown in Fig. 1. The median TI was 4.1 (range, 2.1-9.8) weeks. Based on this observation, we compared the groups based on $\mathrm{TI}<4$ weeks $(\mathrm{n}=66,38.4 \%)$ and TI $\geq 4$ weeks $(n=106,61.6 \%)$. The majority of patients $(n=123$, $71.5 \%$ ) had received chemotherapy within 3 to 5 weeks of surgery, with few patients receiving chemotherapy within 3 weeks or after 8 weeks.

Clinical factors affecting survival outcomes. Following a univariate analysis, the clinical factors that showed a significant effect on the survival outcome included tumor stage and TI. The median DFS for patients with stage III gastric adenocarcinoma was significantly shorter than that for patients with stage II gastric adenocarcinoma [4.3 years vs. not reached $(\mathrm{NR})$, respectively; hazard ratio $(\mathrm{HR})=2.22$, $\mathrm{P}=0.0013$, Fig. 2A]. OS showed a similar statistical trend toward patients with stage II vs. III gastric adenocarcinoma (NR vs. 7.0 years, respectively; HR=2.01, $\mathrm{P}=0.0098$, Fig. 2B). Similarly, a TI of greater than or less than 4 weeks had a significant impact on patients' survival. Patients who started chemotherapy 4 weeks or more after surgery showed a significantly shorter median DFS compared to patients who had started chemotherapy within 4 weeks of surgery (6.0 vs. 8.1 years, respectively; $H R=1.80, P=0.0277$, Fig. $3 \mathrm{~A}$ ). The median OS in patients with $\mathrm{TI} \geq 4$ weeks was also shorter than that in patients with TI $<4$ weeks (7.0 vs. NR years, $\mathrm{HR}=2.15, \mathrm{P}=0.0133$, Fig. 2B). Other clinical factors such as sex, age, and whether the planned chemotherapy was completed had no significant effect on either DFS or OS (Table II). After adjusting for the effect of tumor stage in the multivariate analysis, $\mathrm{TI} \geq 4$ weeks still showed a significantly worse effect on reducing both DFS and OS (DFS: HR=1.737, $\mathrm{P}=0.040$; OS: $\mathrm{HR}=1.939, \mathrm{P}=0.018$, Table III). In addition to the median value of TI (4 weeks), we also compared DFS and OS based on a different TI from 3 to 8 weeks. However, only a TI of 4 weeks discriminated between DFS and OS.

Reasons for delayed adjuvant chemotherapy. Reasons for delays in the administration of chemotherapy included postoperative complications (i.e., intra-abdominal abscesses, anastomotic site leakage, paralytic ileus, or wound infections), inadequate physical condition to start chemotherapy (i.e., general weakness or poor oral intake), and others (i.e., low patient compliance, economic status, or delays due to patient's unavailability). Considering the retrospective nature of this study, obtaining the reasons for delaying AC was challenging. However, the most common causes for delays of longer than 4 weeks in administering AC were surgical complications $(n=29 / 105,26.6 \%)$, followed by a poor general condition by the patient $(n=13 / 105,12.3 \%)$. All reasons for delayed AC are listed in Table IV. For surgical complications, the types of complications were investigated in more detail (Supplementary Table). The median DFS of patients who experienced surgical complication was insignificantly shorter than that of patients without surgical complication (7.3 vs. 8.1 years, $\mathrm{HR}=1.34, \mathrm{P}=0.326$ ). The median $\mathrm{OS}$ also tended to be numerically shorter without statistical significance in patients who experienced postoperative complications than that in patients who did not, although the median values in both groups were not reached $(\mathrm{HR}=1.52, \mathrm{P}=0.185)$.

\section{Discussion}

AC is typically administered to eradicate residual cancer and invisible micrometastases that may remain after surgery. Previous studies using animal models have shown that surgical resection of primary tumors increased the number of circulating tumor cells and promoted the proliferation of residual cells (13). Surgery has also been shown to promote the production of oncogenic growth factors such as transforming growth factor- $\alpha$ and to significantly reduce the immunotherapeutic effect of interleukin-2 and lymphokine-activated killer cells (14). Cellular proliferation of cancer cells progresses rapidly initially and then progressively. Therefore, when the tumor burden is minimal following surgery, it is expected that $\mathrm{AC}$ should be administered as soon as possible. However, a significantly early AC start can affect the patient's recovery such as wound healing and may cause adverse effects when the patient's general condition has not been fully recovered. Conversely, beginning AC significantly late can increase the risk of recurrence due to regrowth of microscopic or indolent foci of viable tumor cells. As a result, several studies have evaluated the optimal timing for $\mathrm{AC}$ administration, particularly in patients with colon or breast cancer.

In colon cancer, early $\mathrm{AC}$ administration has been shown to improve OS compared to late administration, using various time points from 60 days to 8 weeks $(15,16)$. Another study showed that AC started within 4 to 8 weeks improved survival compared to later starts of longer than 8 weeks (17). A previous meta-analysis has also shown that relative OS decreases by $14 \%$ for every 4 -week delay in the initiation of AC (18). Early AC within 20 days improved DFS, whereas initiation of AC within 21 days of surgery was not associated with OS or DFS in patients with early breast cancer, suggesting there is some complexity and ambiguity in the optimal time of AC after tumor resection surgery $(19,20)$. Similar reports in gastric cancer are insufficient; thus, the appropriate timing of $\mathrm{AC}$ administration in patients with gastric cancer is yet to be established. In a Korean retrospective study, AC within 28 days led to significant improvement in 10-year OS, suggesting the early initiation of AC after gastrectomy (21). However, the 

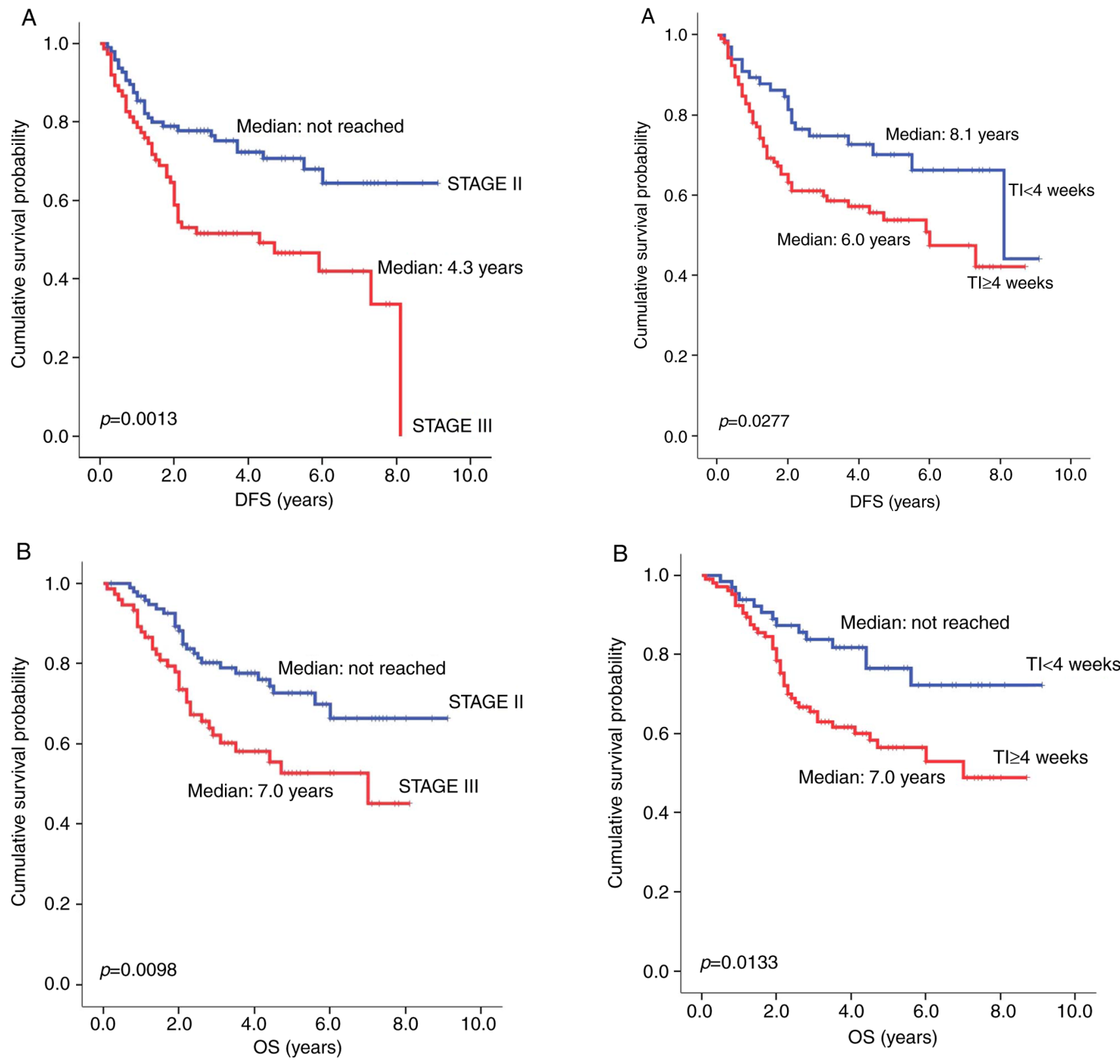

Figure 2. (A) Disease-free survival and (B) overall survival according to staging, II vs. III.

chemotherapeutic agents in the study (5-FU, mitomycin C, and polysaccharide-K) are not the standard agents used recently. Another Korean study showed AC administered within 8 weeks instead of 4 weeks of surgery improved survival outcomes (22). Interestingly, the results of the analysis of subgroups who were able to start AC within 4 weeks because of minimally invasive surgery (i.e., laparoscopic or robot-assisted gastrectomy) showed a significantly better OS and relapse-free survival compared to subgroups who started AC after 4 weeks. Conversely, other reports showed no survival benefit for patients who received AC within 4 weeks of surgery (23). A study in Taiwan showed that starting AC within 8 weeks of a gastrectomy resulted in an improved 5-year recurrent-free survival rate, possibly contributing to an improved OS (24). Another study on AC using S-1 chemotherapy, from Japan, found that the timing of $\mathrm{AC}$ was not associated with OS (25).

Figure 3. (A) Disease-free survival and (B) overall survival according to the time interval, less than 4 weeks compared to 4 weeks or more.

However, others reported that S-1 administration within 6 weeks of surgery was associated with a decrease in recurrence rates and an increase in survival time (26). A recent meta-analysis showed a survival benefit when $\mathrm{AC}$ was started within 6 to 8 weeks of surgery. However, when $A C$ was started after 8 weeks, a $20 \%$ increased risk of death was observed (27). Notwithstanding, this meta-analysis did not evaluate solely gastric cancer, but also included other types of cancer such as colorectal or pancreatic cancer. Taken together, it is important to set an optimal TI for AC delivery, despite differences in patient's recovery after surgery, surgery methods, types of AC, and tumor patterns. It has been not exactly known why the results of previous studies are inconsistent with each other. Considering that prospective comparative studies have not yet been conducted, it is inevitable to interpret them in consideration of the number of samples, methods of statistical analysis, and potential biases in each study. However, as reported in the 
Table II. Univariate analysis of disease-free survival and overall survival based on clinical factors.

\begin{tabular}{|c|c|c|c|c|c|}
\hline \multirow[b]{2}{*}{ Variable } & \multirow[b]{2}{*}{$\mathrm{N}$} & \multicolumn{2}{|l|}{ DFS } & \multicolumn{2}{|l|}{ OS } \\
\hline & & HR $(95 \% \mathrm{CI})$ & P-value & HR $(95 \% \mathrm{CI})$ & P-value \\
\hline Age & & & 0.3672 & & 0.7545 \\
\hline$<60$ & 88 & 1 & & 1 & \\
\hline$\geq 60$ & 84 & $1.56(0.97-2.53)$ & & $1.35(0.80-2.29)$ & \\
\hline Sex & & & 0.657 & & 0.4181 \\
\hline Male & 124 & 1 & & 1 & \\
\hline Female & 48 & $1.60(0.97-2.53)$ & & $1.27(0.72-2.24)$ & \\
\hline Stage & & & $0.0013^{\mathrm{a}}$ & & 0.0098 \\
\hline II & 97 & 1 & & 1 & \\
\hline III & 75 & $2.22(1.37-3.60)$ & & $2.01(1.18-3.40)$ & \\
\hline \multicolumn{6}{|l|}{ Type of adjuvant chemotherapy } \\
\hline XELOX & 48 & 1 & & 1 & \\
\hline TS-1 & 86 & $0.60(0.34-1.08)$ & 0.0872 & $0.65(0.34-1.27)$ & 0.2059 \\
\hline FP & 24 & $1.29(0.65-2.57)$ & 0.4723 & $1.67(0.80-3.49)$ & 0.1760 \\
\hline TS-1/cisplatin & 6 & $0.90(0.27-3.00)$ & 0.8594 & $1.34(0.41-4.46)$ & 0.6303 \\
\hline FOLFOX & 5 & $0.86(0.22-3.33)$ & 0.8315 & $0.65(0.11-3.85)$ & 0.6330 \\
\hline Doxifluridine & 3 & $0.42(0.03-6.15)$ & 0.5274 & $0.55(0.03-9.67)$ & 0.6798 \\
\hline Planned adjuvant chemotherapy was completed & & & 0.0969 & & 0.2675 \\
\hline No & 98 & 1 & & 1 & \\
\hline Yes & 74 & $1.50(0.93-2.43)$ & & $1.35(0.80-2.28)$ & \\
\hline Time interval & & & 0.0277 & & 0.0133 \\
\hline$<4$ weeks & 67 & 1 & & 1 & \\
\hline$\geq 4$ weeks & 105 & $1.8(1.067-3.045)$ & & $2.15(1.173-3.939)$ & \\
\hline
\end{tabular}

${ }^{\text {a}}$ Firth's penalized maximum likelihood bias reduction method for Cox regression was used. DFS, disease-free survival; OS, overall survival; HR, hazard ratio; CI, confidence interval; XELOX, oxaliplatin plus capecitabine; FP, cisplatin plus 5-fluorouracil; FOLFOX, oxaliplatin plus leucovorin plus 5-fluorouracil; NA, not applicable.

Table III. Multivariate analysis of DFS and OS based on stage and TI of 4 weeks.

\begin{tabular}{|c|c|c|c|c|}
\hline \multirow[b]{2}{*}{ Variables } & \multicolumn{2}{|l|}{ DFS } & \multicolumn{2}{|l|}{ OS } \\
\hline & $\mathrm{HR}(95 \% \mathrm{CI})$ & P-value & $\mathrm{HR}(95 \% \mathrm{CI})$ & P-value \\
\hline Stage (III vs. II) & $2.163(1.331-3.515)$ & 0.002 & $1.939(1.143-3.290)$ & 0.014 \\
\hline Time interval ( $<4$ weeks vs. $\geq 4$ weeks) & $1.737(1.026-2.939)$ & 0.040 & $1.939(1.143-3.290)$ & 0.018 \\
\hline
\end{tabular}

DFS, disease-free survival; OS, overall survival; HR, hazard ration; CI, confidential interval; TI, time interval.

aforementioned study (22) that early $\mathrm{AC}$ within 4 weeks due to minimally invasive surgical technique led a better survival outcomes, it may be reasonable to recommend starting $\mathrm{AC}$ as early as possible (i.e., within 4 weeks) as long as the patient's condition improves sufficiently after surgery and there is no reason to delay the administration of $\mathrm{AC}$.

In this study, we dichotomized the TI as less than vs. equal or more than 4 weeks based on the median value of TI within our study population (4.1 months). Additionally, we further examined the effect of various TIs set arbitrarily every 1 week to evaluate whether there were significant differences based on the different TIs. We found that OS and DFS were greater in the group with a TI lower than 4 weeks than those in the group with a TI higher than 4 weeks. In addition to TI, TNM stage was another significant factor that affected patients' survival in our multivariate analysis (stage III vs. II, HR=2.22.95\% CI: 1.37-3.60). This is consistent with the results from previous studies $(10,11,20)$ and may suggest that our study population provides a significant representation of the general population in terms of disease characteristics and the natural course of disease, despite the retrospective nature of our cohort. Therefore, the significance of the 4-week TI obtained in this 
Table IV. Reasons for delayed chemotherapy ( $\geq 4$ weeks).

Reason for delayed chemotherapy

Postoperative complications (i.e., intra-abdominal abscess, anastomosis site leakage, ileus, wound infection)

Inadequate condition to start chemotherapy (i.e., general weakness, poor oral intake)

Other conditions requiring hospitalization, excluding postoperative complications

Personal condition (i.e., low patient's compliance, economic status, delayed due to foreign residence)

Total

study may be considered as a reliable TI for the general population as well. Additionally, this study included solely patients who received gastrectomy with D2 lymph node dissection, unlike previous studies that included patients undergoing both D1 and D2 dissections. Since D2 dissection has become a standard technique, our study may be more appropriately applied to the real-world clinical practice $(28,29)$.

Besides its retrospective nature, our study has some limitations. First, preexisting comorbidity data for patients were missing. Comorbidities are important factors to consider as they can affect the recovery from surgery, start of chemotherapy, and patients' long-term survival. We were not able to investigate the effects of comorbidities in this instance. However, as patients included here were sufficiently healthy to withstand a radical surgery, even if other comorbidities were observed, it is possible to assume that the overall health status of our patients was satisfactory. In addition to the comorbidities that had already been diagnosed, it has been found that postoperative complications have a significantly negative impact on survival, including both on OS and disease-specific mortality, in patients with gastric cancer (30). In fact, when comparing HR, there was a tendency of worse long-term survival outcomes according to the immediate postoperative complications. The fact that statistical significance was not observed in this study population might be related to the insufficient sample size. Second, although the Korean study mentioned earlier showed the association between the possibility of early $\mathrm{AC}$ and minimally invasive surgical procedures, we did not investigate the surgical methods used. A study reported that a laparoscopic approach was associated with a reduced recovery time and allowed for a shortened TI to AC administration (31). Other studies have shown that this approach was not associated with the time of AC start and that although it allowed for earlier discharge following surgery, its benefits did not last longer than in open surgery post-discharge (23). Third, 98 of the 172 patients (57\%) did not complete the planned AC. However, these patients were equally distributed in both groups of patients with TI $\geq 4$ weeks and $<4$ weeks; hence, the potential effect might be offset. Finally, a selection bias may be present in our data considering that this study was conducted in a single center and with a small number of patients.

In conclusion, this study suggests that $\mathrm{AC}$ should be initiated within 4 weeks of surgery with D2 resection in patients with gastric cancer. Delays longer than 4 weeks in $\mathrm{AC}$ administration for any reason may be detrimental to patients' survival.

\section{Acknowledgements}

The current study was presented at European Society for Medical Oncology (ESMO) Asia 2018 Congress, 23 Nov-25 Nov 2018 in Singapore, and published as abstract no. 1199 in Annals of Oncology (2018) 29 (suppl_9): ix46-ix66. 10.1093/annonc/mdy432.

\section{Funding}

No funding was received.

\section{Availability of data and material}

The datasets used and/or analyzed during the current study are available from the corresponding author on reasonable request.

\section{Authors' contributions}

CHM designed the study. GTA and CHM collected patient data and performed the initial statistical analysis. SJJ performed the detailed statistical analysis. GTA was a major contributor in writing the manuscript. CHM supervised the written manuscript. SKB, HJK and JJH provided the clinical information for patients included in the analysis. SKB, HJK and JJH interpreted the results and wrote the first draft of the discussion section. All authors read and approved the final manuscript. All authors read and approved the final manuscript.

\section{Ethics approval and consent to participate}

The current study was approved by Kyung Hee University Hospital IRB (approval no. KHUH 2020-01-044).

\section{Patient consent for publication}

Not applicable.

\section{Competing interests}

The authors declare that they have no competing interests.

\section{References}

1. Torre LA, Bray F, Siegel RL, Ferlay J, Lortet-Tieulent J and Jemal A: Global cancer statistics, 2012. CA Cancer J Clin 65: 87-108, 2015. 
2. Gunderson LL: Gastric cancer-patterns of relapse after surgical resection. Semin Radiat Oncol 12: 150-161, 2002.

3. D'Angelica M, Gonen M, Brennan MF, Turnbull AD, Bains M and Karpeh MS: Patterns of initial recurrence in completely resected gastric adenocarcinoma. Ann Surg 240: 808-816, 2004.

4. Noh SH, Park SR, Yang HK, Chung HC, Chung IJ, Kim SW, Kim HH, Choi JH, Kim HK, Yu W, et al: Adjuvant capecitabine plus oxaliplatin for gastric cancer after D2 gastrectomy (CLASSIC): 5-year follow-up of an open-label, randomised phase 3 trial. Lancet Oncol 15: 1389-1396, 2014.

5. Sakuramoto S, Sasako M, Yamaguchi T, Kinoshita T, Fujii M, Nashimoto A, Furukawa H, Nakajima T, Ohashi Y, Imamura $\mathrm{H}$, et al: Adjuvant chemotherapy for gastric cancer with S-1, an oral fluoropyrimidine. N Engl J Med 357: 1810-1820, 2007.

6. Becerra AZ, Aquina CT, Mohile SG, Tejani MA, Schymura MJ, Boscoe FP, Xu Z, Justiniano CF, Boodry CI, Swanger AA, et al: Variation in delayed time to adjuvant chemotherapy and disease-specific survival in stage III colon cancer patients. Ann Surg Oncol 24: 1610-1617, 2017.

7. Chavez-MacGregor M, Clarke CA, Lichtensztajn DY and Giordano SH: Delayed initiation of adjuvant chemotherapy among patients with breast cancer. JAMA Oncol 2: 322-329, 2016.

8. Japanese Gastric Cancer Association: Japanese gastric cancer treatment guidelines 2014 (ver. 4). Gastric Cancer 20: 1-19, 2017.

9. Guideline Committee of the Korean Gastric Cancer Association (KGCA), Development Working Group \& Review Panel: Korean Practice Guideline for gastric cancer 2018: An evidence-based, multi-disciplinary approach. J Gastric Cancer 19: 1-48, 2019.

10. Sasako M, Sakuramoto S, Katai H, Kinoshita T, Furukawa H, Yamaguchi T, Nashimoto A, Fujii M, Nakajima T and Ohashi Y: Five-year outcomes of a randomized phase III trial comparing adjuvant chemotherapy with S-1 versus surgery alone in stage II or III gastric cancer. J Clin Oncol 29: 4387-4393, 2011.

11. Bang YJ, Kim YW, Yang HK, Chung HC, Park YK, Lee KH, Lee KW, Kim YH, Noh SI, Cho JY, et al: Adjuvant capecitabine and oxaliplatin for gastric cancer after D2 gastrectomy (CLASSIC): A phase 3 open-label, randomised controlled trial. Lancet 379: 315-321, 2012.

12. Bang YJ, Van Cutsem E, Fuchs CS, Ohtsu A, Tabernero J, Ilson DH, Hyung WJ, Strong VE, Goetze TO, Yoshikawa T, et al KEYNOTE-585: Phase III study of perioperative chemotherapy with or without pembrolizumab for gastric cancer. Future Oncol 15: 943-952, 2019.

13. Gunduz N, Fisher B and Saffer EA: Effect of surgical removal on the growth and kinetics of residual tumor. Cancer Res 39: 3861-3865, 1979.

14. Ono I, Gunji H, Suda K, Iwatsuki K and Kaneko F: Evaluation of cytokines in donor site wound fluids. Scand J Plast Reconstr Surg Hand Surg 28: 269-273, 1994.

15. Bayraktar UD, Chen E, Bayraktar S, Sands LR, Marchetti F, Montero AJ and Rocha-Lima CM: Does delay of adjuvant chemotherapy impact survival in patients with resected stage II and III colon adenocarcinoma? Cancer 117: 2364-2370, 2011

16. Des Guetz G, Nicolas P, Perret GY, Morere JF and Uzzan B: Does delaying adjuvant chemotherapy after curative surgery for colorectal cancer impair survival? A meta-analysis. Eur J Cancer 46: 1049-1055, 2010.

17. Klein M, Azaquoun N, Jensen BV and Gögenur I: Improved survival with early adjuvant chemotherapy after colonic resection for stage III colonic cancer: A nationwide study. J Surg Oncol 112: 538-543, 2015.

18. Biagi JJ, Raphael MJ, Mackillop WJ, Kong W, King WD and Booth CM: Association between time to initiation of adjuvant chemotherapy and survival in colorectal cancer: A systematic review and meta-analysis. JAMA 305: 2335-2342, 2011.
19. Colleoni M, Bonetti M, Coates AS, Castiglione-Gertsch M, Gelber RD, Price K, Rudenstam CM, Lindtner J, Collins J, Thürlimann B, et al: Early start of adjuvant chemotherapy may improve treatment outcome for premenopausal breast cancer patients with tumors not expressing estrogen receptors. The International Breast Cancer Study Group. J Clin Oncol 18: 584-590, 2000.

20. Shannon C, Ashley S and Smith IE: Does timing of adjuvant chemotherapy for early breast cancer influence survival? J Clin Oncol 21: 3792-3797, 2003

21. Kang SY, Ahn MS, Song GW, Choi YW, Lee HW, Jeong SH, Park JS, Cho YK, Han SU, Sheen SS, et al: Does the timing of adjuvant chemotherapy for gastric cancer influence patient outcome? Acta Oncol 54: 1231-1234, 2015.

22. Park HS, Jung M, Kim HS, Kim HI, An JY, Cheong JH, Hyung WJ, Noh SH, Kim YI, Chung HC and Rha SY: Proper timing of adjuvant chemotherapy affects survival in patients with stage 2 and 3 gastric cancer. Ann Surg Oncol 22: 224-231, 2015.

23. Lee Y, Min SH, Park KB, Park YS, Kim JW, Ahn SH, Kim JW, Park DJ, Lee KW and Kim HH: Effect of early adjuvant chemotherapy on survival of advanced gastric cancer patients: A propensity score-matched analysis. J Gastric Cancer 18: 58-68, 2018.

24. Huang SM, Chen YC, Chen WY, Yang LY, Tsan DL, Tsang NM, Yap WK, Tsai CS, Leung WM, Hong JH, et al: Optimal timing for postsurgical adjuvant therapy in patients with gastric cancer: A propensity score matching study. J Cancer 10: 332-340, 2019.

25. Fujitani K, Kurokawa Y, Takeno A, Endoh S, Ohmori T, Fujita J, Yamasaki M, Takiguchi S, Mori M and Doki Y; Osaka University Clinical Research Group for Gastroenterological Surgery: Time to initiation or duration of S-1 adjuvant chemotherapy; which really impacts on survival in stage II and III gastric cancer? Gastric Cancer 21: 446-452, 2018.

26. Yamamoto M, Sakaguchi Y, Kinjo N, Yamaguchi S, Egashira A Minami K, Ikeda Y, Morita M, Toh Y and Okamura T: S-1 adjuvant chemotherapy earlier after surgery clinically correlates with prognostic factors for advanced gastric cancer. Ann Surg Oncol 23: 546-551, 2016.

27. Petrelli F, Zaniboni A, Ghidini A, Ghidini M, Turati L, Pizzo C, Ratti M, Libertini $\mathrm{M}$ and Tomasello G: Timing of adjuvant chemotherapy and survival in colorectal, gastric, and pancreatic cancer. A systematic review and meta-analysis. Cancers (Basel) 11: 550, 2019.

28. Degiuli M, Sasako M, Calgaro M, Garino M, Rebecchi F, Mineccia M, Scaglione D, Andreone D, Ponti A and Calvo F; Italian Gastric Cancer Study Group: Morbidity and mortality after D1 and D2 gastrectomy for cancer: Interim analysis of the Italian Gastric Cancer Study Group (IGCSG) randomised surgical trial. Eur J Surg Oncol 30: 303-308, 2004.

29. Degiuli M, Sasako M, Ponti A and Calvo F: Survival results of a multicentre phase II study to evaluate D2 gastrectomy for gastric cancer. Br J Cancer 90: 1727-1732, 2004.

30. Kubota T, Hiki N, Sano T, Nomura S, Nunobe S, Kumagai K, Aikou S, Watanabe R, Kosuga T and Yamaguchi T: Prognostic significance of complications after curative surgery for gastric cancer. Ann Surg Oncol 21: 891-898, 2014.

31. Kaito A, Kinoshita T, Shitara K, Shibasaki H and Nishida T: Timing of initiation of adjuvant chemotherapy for gastric cancer: A case-matched comparison study of laparoscopic vs. open surgery. Eur J Surg Oncol 43: 801-807, 2017.

This work is licensed under a Creative Commons Attribution-NonCommercial-NoDerivatives 4.0 International (CC BY-NC-ND 4.0) License. 